\title{
PERFIL EPIDEMIOLÓGICO DA POPULAÇÃO SUBMETIDA À REVASCULARIZAÇÃO CARDÍACA E ACESSO AO SISTEMA ÚNICO DE SAÚDE
}

\author{
Cintia Koerich ${ }^{1}$, Gabriela Marcellino de Melo Lanzoni², Betina Hörner Schlindwein Meirelles², Maria \\ Aparecida Baggio ${ }^{3}$, Giovana Dorneles Callegaro Higashi ${ }^{4}$, Alacoque Lorenzini Erdmann ${ }^{5}$
}

\begin{abstract}
RESUMO: O objetivo deste estudo foi descrever o perfil epidemiológico de uma população submetida à revascularização cardíaca e compreender como esta população acessa os serviços de saúde para promoção, prevenção, tratamento e reabilitação. Estudo descritivo, transversal, amostra de 99 participantes, entre março de 2013 e fevereiro de 2014. Em relação ao perfil, 70 (70,7\%) homens, 61 (61,6\%) casados, 53 (53,5\%) aposentados, $86(86,9 \%)$ procedentes da região metropolitana, com idade média de $61,3( \pm 8,5)$ e média de anos de estudos de seis $( \pm 3,4)$, as comorbidades prevalentes foram Hipertensão Arterial Sistêmica $84(84,8 \%)$ e Diabetes Mellitus 42 (42,4\%). Referente ao Acesso aos serviços de saúde, 49 (49,4\%) acessaram o hospital e 31 (31,3\%) a Unidade Básica de Saúde. Observa-se a necessidade de ações estratégicas que facilitem o acesso e acompanhamento desta população aos serviços de saúde, em especial na Atenção primária à saúde, antes e após a revascularização cardíaca.
\end{abstract}

DESCRITORES: Perfil de Saúde; Acesso aos Serviços de Saúde; Sistema Único de Saúde; Cirurgia Torácica; Enfermagem.

\section{EPIDEMIOLOGICAL PROFILE OF THE POPULATION WHO UNDERWENT MYOCARDIAL REVASCULARIZATION AND ACCESS TO THE UNIFIED HEALTH SYSTEM}

ABSTRACT: The present study aimed to describe the epidemiological profile of a population who underwent myocardial revascularization surgery and gain insight on how this population accesses the health services for promotion, prevention, treatment and rehabilitation. Descriptive cross-sectional study with a sample of 99 participants conducted between March 2013 and February 2014. Concerning the profile of the participants, 70 (70.7\%) were men, $61(61.6 \%)$ were married, $53(53.5 .9 \%)$ were retired, $86(86.9 \%)$ lived in the Metropolitan region and had an average age of $61.3( \pm 8.5)$ and a mean schooling of six years $( \pm 3.4)$. The prevalent comorbidities were Systemic Arterial Hypertension, 84 (84.8\%) and Diabetes Mellitus, 42 (42.4\%). Regarding access to health services, $49(49.4 \%)$ were admitted to a hospital and $31(31.3 \%)$ were assisted at a Basic Health Unit (BHU). Strategic actions aimed to facilitate access of this population to health services and their monitoring, especially in Primary Health Care (PHC), before and after myocardial revascularization surgery, is needed.

DESCRIPTORS: Health Profile; Access to Health Services; Unified Health System; Thoracic surgery; Nursing.

\section{PERFIL EPIDEMIOLÓGICO DE LA POBLACIÓN SOMETIDA A LA REVASCULARIZACIÓN CARDÍACA Y ACCESO AL SISTEMA ÚNICO DE SALUD}

RESUMEN: Estudio cuyo intento fue describir el perfil epidemiológico de una población sometida a revascularización cardíaca y comprender como esa población tiene acceso a los servicios de salud para promoción, prevención, tratamiento y rehabilitación. Estudio descriptivo, transversal, con 99 participantes, realizado entre marzo de 2013 y febrero de 2014. Acerca de las constataciones sobre el perfil, fueron $70(70,7 \%)$ hombres, 61 (61,6\%) casados, $53(53,5 \%)$ jubilados, $86(86,9 \%)$ que vinieron de la región metropolitana, con edad media de $61,3( \pm 8,5)$ y media de años de estudios de seis $( \pm 3,4)$, las comorbilidades prevalentes fueron Hipertensión Arterial Sistémica $84(84,8 \%)$ y Diabetes Mellitus 42 (42,4\%). Acerca del acceso a los servicios de salud, 49 (49,4\%) fueron al hospital y 31 (31,3\%) a la Unidad Básica de Salud. Se observa la necesidad de acciones estratégicas que faciliten el acceso y el acompañamiento de esa población a los servicios de salud, principalmente en la Atención básica a la salud, antes y después de la revascularización cardíaca. DESCRIPTORES: Perfil de Salud; Acceso a los Servicios de Salud; Sistema Único de Salud; Cirugía Torácica; Enfermería.

${ }^{1}$ Enfermeira. Doutoranda em Enfermagem da Universidade Federal de Santa Catarina. Florianópolis, SC, Brasil.

${ }^{2}$ Enfermeira. Doutora em Enfermagem. Docente de Enfermagem da Universidade Federal de Santa Catarina. Florianópolis, SC, Brasil.

${ }^{3}$ Enfermeira. Doutora em Enfermagem. Docente de Enfermagem da Universidade Estadual do Oeste do Paraná. Cascavel, PR, Brasil.

${ }^{4}$ Enfermeira. Pós-Doutoranda em Enfermagem da Universidade Federal de Santa Catarina. Florianópolis, SC, Brasil.

${ }^{5}$ Enfermeira. Doutora em Filosofia da Enfermagem. Docente de Enfermagem da Universidade Federal de Santa Catarina. Florianópolis, SC, Brasil.

Autor Correspondente:

Recebido: $21 / 02 / 2017$

Cintia Koerich

Universidade Federal de Santa Catarina

Finalizado: 19/08/2017

R. Deputado Walter Gomes, 580 - 88050-500 - Florianópolis, SC, Brasil

E-mail: Cintia.koerich@gmail.com 


\section{- INTRODUÇÃO}

As doenças cardiovasculares (DCV) estão entre as principais causas de morbimortalidade no Brasil e no mundo, sendo sua causa multifatorial, evidenciada pelo perfil epidemiológico, fatores hereditários e o convívio com fatores de riscos $^{(1)}$. As DCV podem ser tratadas clínica ou cirurgicamente. No tratamento cirúrgico, a cirurgia de revascularização do miocárdio (CRM) é considerada procedimento consolidado no tratamento das doenças isquêmicas do coração ${ }^{(2)}$.

No entanto, considerando que a gravidade dos pacientes submetidos à intervenção cirúrgica cardíaca, em especial à CRM, tem aumentado consideravelmente nas últimas décadas, ampliando o risco de complicações e mortalidade, são crescentes as propostas de ações de prevenção, tratamento e reabilitação das DCV na agenda de saúde ${ }^{(2)}$.

O Sistema Único de Saúde (SUS) prevê a partir da Constituição Federal de 1988 a universalidade do acesso, da integralidade das práticas e dos processos e da equidade na distribuição dos diferentes recursos. O acesso, neste caso, é entendido como um conceito ampliado de atenção resolutiva às necessidades de saúde de uma determinada população e território ${ }^{(3)}$.

O acesso oportuno e a continuidade do cuidado são consideradas medidas operacionais empregadas internacionalmente para verificar a qualidade do acesso à atenção primária à saúde (APS). O acesso oportuno se refere à capacidade do serviço de atender o usuário em suas necessidades de saúde, evitando encaminhamentos aos serviços de emergência e maiores custos. A continuidade do cuidado é a oportunidade do usuário ter acesso ao profissional de saúde de referência para acompanhamento sempre que necessário, promovendo o vínculo e o cuidado individualizado(4). A limitação do acesso aos serviços e acompanhamento à saúde tem como consequência o agravamento ou cronicidade do problema de saúde ${ }^{(5-6)}$.

Neste contexto, torna-se importante na atuação do enfermeiro e de outros profissionais de saúde, reconhecer o perfil epidemiológico do paciente que realiza CRM e as necessidades de saúde desta população para que o cuidado de enfermagem possa ser realizado de forma criativa, humana e interativa, buscando a integralidade do ser humano, promovendo o autocuidado ${ }^{(7)}$.

Vários estudos têm apresentado o perfil epidemiológico dos pacientes submetidos à CRM, no entanto, este estudo buscou apresentar um perfil ampliado considerando dados sobre o acesso dessa população aos serviços de saúde, para acompanhamento antes e após o diagnóstico de DCV.

Assim, questiona-se: Qual o perfil epidemiológico de uma população submetida à revascularização cardíaca em uma instituição de referência cardiovascular para o estado de Santa Catarina e como esta população acessa os serviços no Sistema Único de Saúde para acompanhamento de saúde? O objetivo deste estudo foi descrever o perfil epidemiológico de uma população submetida à revascularização cardíaca e compreender como esta população acessa os serviços de saúde para promoção, prevenção, tratamento e reabilitação.

\section{METODOLOGIA}

Trata-se de estudo descritivo, prospectivo, com delineamento transversal desenvolvido em um hospital público referência cardiovascular no sul do Brasil. A coleta dos dados foi realizada em duas fases.

Na primeira fase, a amostra foi constituída de 99 pacientes, sendo critério de inclusão estar internado na instituição aguardando a CRM e critério de exclusão não estar em condições clínicas para responder o questionário. O número de pacientes que realizou CRM, no período do estudo, de março de 2013 a fevereiro de 2014, foi de 110. Destes, 11 pacientes não aceitaram ou contemplavam os critérios de exclusão para participar do estudo, resultando na perda amostral de $10 \%$. Foi utilizado questionário estruturado para coleta de dados. Informações que não eram de conhecimento do participante foram coletadas em prontuário, com a devida autorização.

Para análise dos dados, na primeira fase, foram verificadas as frequências e os percentuais para as 
medidas qualitativas e as médias para as medidas quantitativas, sendo consideradas as variáveis de Identificação e Sociodemográficas (idade, sexo, estado civil, procedência, escolaridade, ocupação); de Comorbidades Associadas à Doença Arterial Coronariana (DAC) - (hipertensão arterial sistêmica (HAS), diabetes mellitus (DM), doença pulmonar obstrutiva crônica (DPOC), doença cerebrovascular, insuficiência renal e outras); de Fatores de Risco para DAC (pressão alta, glicemia elevada, dislipidemia, tabagismo, antecedentes familiares, e outras); e de Acesso aos serviços de saúde para promoção, prevenção, tratamento e reabilitação (acessa e utiliza a unidade básica de saúde de referência, serviço de saúde procurado quando precisa de atendimento, local de realização de exames diagnósticos, tratamento medicamentoso realizado para doença cardiovascular, acessa a terapia medicamentosa na rede pública, participa de grupos ou atividade de saúde na unidade básica de saúde (UBS) de referência, faz reabilitação cardíaca (para quem já é revascularizado).

Na segunda fase, a amostra foi aleatória e teve participação de oito pacientes que compuseram a primeira fase. As entrevistas ocorreram nos meses de março e abril de 2014, com uso de questionário semiestruturado para compreender com maior detalhamento aspectos prevalentes na primeira fase do estudo. Para a análise dos dados, foi utilizada a Análise Temática de Conteúdo, que inclui processo sistemático de pré-análise, exploração do material, tratamento dos resultados e interpretação dos $\operatorname{dados}^{(8)}$.

A primeira etapa ou pré-análise compreendeu a aproximação e organização do material ${ }^{(8)}$, a formulação e reformulação de hipóteses e objetivos. A segunda etapa, de exploração do material, incluiu a classificação dos dados, objetivando a compreensão essencial do texto ${ }^{(8)}$. Os dados brutos foram codificados, reduzindo o texto a palavras ou expressões significativas. Reformularam-se os agrupamentos de dados segundo o tema de acordo com a similaridade e diferença de ideias. Na terceira etapa, denominada análise temática, ocorreu o tratamento dos resultados obtidos e a interpretação dos dados ${ }^{(8)}$. A saturação dos dados foi obtida com a ausência de novos elementos relevantes. Os dados foram organizados no programa NVivo 10.

O estudo faz parte de um macroprojeto intitulado "Paciente cardíaco revascularizado: processo de referência e contrarreferência dos serviços de saúde de Santa Catarina" aprovado pelo Comitê de Ética em Pesquisa com Seres Humanos da Universidade Federal de Santa Catarina (CEPSH/UFSC) sob o número 120.184 em 2012. Com o intuito de preservar a identidade dos participantes, as falas foram identificadas pela letra "E" seguida de um número ordinal correspondente à ordem da entrevista.

\section{- RESULTADOS}

As variáveis de Identificação e Sociodemográficas mostram que dentre os participantes do estudo, $70(70,7 \%)$ eram homens, 61 (61\%) casados, aposentados $53(53,5 \%)$, sendo a maior parte da amostra procedente da região metropolitana do Estado 86 (86,9\%). Quando considerada a ocupação anterior à aposentadoria, 14 (14,1\%) eram pedreiros, 11 (11,1\%) donas de casa e 11 (11,1\%) prestadores de serviços gerais, as outras atividades apresentaram menos que $10 \%$. A média de idade dos participantes foi de $61,3( \pm 8,5)$ anos e a média de anos de estudos foi de seis $( \pm 3,4)$, conforme Tabela 1.

As Comorbidades Associadas à DAC prevalentes foram a HAS em 84 (84,8\%) participantes e a DM em 42 (42,4\%). Os Fatores de Risco para DAC com maior expressão foram pressão arterial sistêmica em 85 pacientes $(85,9 \%)$, antecedente familiar em $65(65,7 \%)$ e dislipidemia em $63(63,6 \%)$, apresentados na Tabela 1.

Tabela 1 - Características de identificação e sociodemográficas, comorbidades associadas e fatores de risco à DAC. Florianópolis, SC, Brasil, 2014 ( $\mathrm{n}=99$ ) (continua)

\begin{tabular}{lll} 
Características & n & \% \\
\hline Sexo & & \\
\hline Feminino & 29 & 29,3 \\
\hline Masculino & 70 & 70,7 \\
\hline
\end{tabular}




\begin{tabular}{|c|c|c|}
\hline \multicolumn{3}{|l|}{ Estado Civil } \\
\hline Solteiro & 8 & 8,1 \\
\hline Casado & 61 & 61,6 \\
\hline Viúvo & 15 & 15,2 \\
\hline Divorciado & 15 & 15,2 \\
\hline \multicolumn{3}{|l|}{ Procedência } \\
\hline Grande Florianópolis & 86 & 86,9 \\
\hline Vale do Itajaí & 1 & 1 \\
\hline Sul & 6 & 6,1 \\
\hline Planalto & 1 & 1 \\
\hline Não informaram & 5 & 5 \\
\hline \multicolumn{3}{|l|}{ Ocupação } \\
\hline Autônomo & 21 & 21,2 \\
\hline Empregado com carteira assinada & 11 & 11,1 \\
\hline Desempregado & 3 & 3 \\
\hline Aposentado & 53 & 53,5 \\
\hline Não informaram & 11 & 11,2 \\
\hline \multicolumn{3}{|l|}{ Idade } \\
\hline Menos de 50 anos & 8 & 8 \\
\hline 50 a 60 anos & 40 & 40,4 \\
\hline 61 a 70 anos & 36 & 36,3 \\
\hline 71 a 80 anos & 13 & 13,1 \\
\hline Mais de 80 anos & 2 & 2 \\
\hline \multicolumn{3}{|l|}{ Anos de estudo } \\
\hline Menos de 1 ano & 5 & 5 \\
\hline De 1 a 3 anos & 10 & 10,1 \\
\hline De 4 a 6 anos & 47 & 47,4 \\
\hline De 7 a 9 anos & 20 & 20,2 \\
\hline Mais de 9 anos & 17 & 17,1 \\
\hline \multicolumn{3}{|l|}{ Comorbidades associadas à DAC +} \\
\hline Hipertensão Arterial Sistêmica & 84 & 84,8 \\
\hline Diabetes Mellitus & 42 & 42,4 \\
\hline Insuficiência Renal & 10 & 10,1 \\
\hline Doença cerebrovascular & 9 & 9,1 \\
\hline Doença Pulmonar Obstrutiva & 8 & 8,1 \\
\hline Outras & 16 & 16,1 \\
\hline \multicolumn{3}{|l|}{ Fatores de risco para DACt } \\
\hline Pressão arterial sistêmica elevada & 85 & 85,9 \\
\hline Antecedente familiar & 65 & 65,7 \\
\hline Dislipidemia & 63 & 63,6 \\
\hline Tabagismo & 54 & 54,5 \\
\hline Glicemia elevada & 40 & 40,4 \\
\hline Outros & 18 & 18,1 \\
\hline
\end{tabular}

Fonte: Dados da pesquisa. Legenda: †Doença Arterial Coronariana.

O nível de instrução da população, considerando o pouco tempo de estudo e as profissões com pouca ou nenhuma exigência de formação, pode interferir no conhecimento e na adoção de hábitos de vida saudáveis ao longo da vida. Estes poderiam minimizar o risco de desenvolvimento ou o agravo 
de doenças evitáveis, como hipertensão e diabetes, dislipidemia, entre outras, repercutindo na identificação de sinais e sintomas da DAC. Segue depoimentos:

Esse problema eu tenho há anos, quando eu caminhava, começava a dar dor no peito. Não era forte, era uma dor suportável, achava que era normal. Não pensava que podia ser do coração. (E1)

Começou com um "queimorzinho" no peito, achei que não era nada e fui levando, trabalhando, fazendo as coisas, tudo normal. De repente, foi apertando, ai "enfartei" e não sabia que era "enfarto". (E2)

Referente ao Acesso aos serviços de saúde para promoção, prevenção, tratamento e reabilitação, quando os participantes necessitaram de atendimento de saúde, 49 (49,4\%) acessaram o hospital, 31 $(31,3 \%)$ a UBS, $13(13,1 \%)$ clínicas particulares, três $(3 \%)$ policlínicas e um $(1 \%)$ unidade de pronto atendimento (UPAS), dois (2,2\%) não informaram.

Em relação ao acesso e utilização da UBS referência, 74 (74,7\%) participantes relataram utilizar a UBS para algum atendimento e $86(86,8 \%)$ participantes informaram utilizar exclusivamente o SUS, com realização de todos os exames diagnósticos da DAC na rede pública, enquanto $12(12,1 \%)$ utilizaram também a rede privada, um (1\%) não informou.

Apesar do significativo número de participantes que afirmou acessar os serviços do SUS para realização de consultas ou realização de exames para diagnóstico, os depoimentos revelam não existir acompanhamento regular do estado de saúde na APS, evidenciando a dificuldade no acesso, conforme depoimentos:

Não tinha acompanhamento. Fui algumas vezes na UPA, mas foi esporádico. Antes do problema cardíaco posso dizer que nenhum [referindo-se ao acompanhamento de saúde]. É uma dificuldade, porque você marca uma consulta e é demorada. Aí, a gente vai deixando e não faz. (E3)

[...] vem um médico num dia, no outro dia vem outro. No posto central tem médico todo dia, mas no posto dos bairros é uma vez por semana e não é sempre o mesmo médico. (E4)

A demora em conseguir uma consulta com o clínico geral ou com o especialista da rede de saúde é salientada nos depoimentos. Recorrer aos serviços de pronto atendimento ou ao serviço privado é uma estratégia utilizada para conseguir avaliação médica. Quando a consulta com o especialista acontece no serviço privado, normalmente os exames de alto custo solicitados são encaminhados a partir da APS. Dos participantes que seguiram aguardando a consulta com o especialista no SUS, evidenciou-se que alguns tiveram que se dirigir ao serviço de alta complexidade numa condição de emergência, sem ter conseguido consultar. Seguem os relatos:

No posto, o clínico geral me atendeu e simplesmente disse que meu problema era de cardiologista, me deu uma requisição para marcar no posto a consulta com o cardiologista que seria numa policlínica. Deixei lá essa consulta para marcar. Isso já faz mais de três meses e até agora eu não tive retorno. Nesse meio tempo, deu problema de emergência e tive que vir para cá [hospital] pela emergência e não pela consulta médica. (E5)

Eu consultava no posto, mas as consultas [com o cardiologista], eu pagava particular, porque demorava muito. Mas os exames que eram caros, eu tinha que deixar no posto para marcar. (E6)

Dos participantes do estudo, 71 (71,7\%) relataram realizar tratamento medicamentoso para DCV ou comorbidades associadas e ter acesso à terapia medicamentosa na rede pública de saúde. Embora a maioria dos participantes tenha declarado retirar medicamentos na APS, isso não significa aquisição de $100 \%$ do tratamento, visto que relatam ser comum a falta de medicamentos padronizados nas UBS. Esse fato favorece a interrupção do tratamento medicamentoso e o avanço da DAC, quando consideradas as condições socioeconômicas desses pacientes e a impossibilidade de financiar o tratamento. Conforme depoimento:

Diziam que a prefeitura não tinha estoque, então eles não redistribuíam nos postos. Sempre foi o que me disseram quando não tinha o medicamento. Daí, eu tinha que passar em outro posto para ver se tinha, senão era comprar. (E7)

Em relação às atividades de reabilitação, apenas 11 (11,3\%) participantes relataram integrar grupos 
terapêuticos relacionados à DCV na UBS (hipertensão, diabetes, condicionamento físico). Dos 20 (20,2\%) participantes revascularizados (CRM e angioplastia primária) deste estudo, seis (30,7\%) realizavam reabilitação cardíaca, destes, cinco $(83,3 \%)$ na instituição hospitalar de referência e um $(16,6 \%)$ na UBS.

A carência de estrutura física e de recursos humanos para o acompanhamento do paciente após revascularização na APS repercute na baixa adesão à reabilitação cardiovascular e em dificuldades para adaptação a um novo estilo de vida. Conforme relato:

Tomava uns remédios e com o tempo eu deixei tudo para lá, não fiz o acompanhamento nem da parte física, nem da alimentação. Continuei comendo as mesmas coisas, gordura, feijoadas, churrasquinho, e foi assim, até que de uns três meses para cá, eu venho sentindo dor, mal estar, essa dificuldade de respirar e vim parar aqui [no hospital]. (E8)

O não acompanhamento dos indivíduos acometidos por DCV ou submetidos a CRM na APS contribui para a não aderência ao tratamento e o aparecimento de novos eventos cardiovasculares/ necessidade de nova revascularização cardíaca.

\section{- DISCUSSÃO}

Os resultados corroboram com estudos que analisaram o perfil epidemiológico de pacientes submetidos a CRM, nos quais houve a prevalência do sexo masculino (70,7\%, 67\%, 68,5\%), com média de idade entre 50 e 75 anos $^{(5-7,2)}$. Poucos estudos trazem a maior parte dos participantes que realizaram CRM como sendo mulheres ${ }^{(9)}$ ou com idade inferior a $50 \operatorname{anos}^{(10)}$. Evidenciando uma população masculina e senil.

Ainda, estudos trazem como perfil de pacientes submetidos à CRM a baixa escolaridade, com no máximo primeiro grau completo, ou cerca de cinco anos de estudo(9,11-12), e em condição de aposentadoria ${ }^{(11)}$, normalmente com ocupações anteriores que geravam rendas de até três salários mínimos ou donas de casa, no caso de mulheres ${ }^{(9)}$.

Ao analisar o tempo de chegada do paciente com infarto agudo do miocárdio na emergência, estudo revelou que, mesmo com uma população com poucos anos de estudo, o reconhecimento dos sinais e dos sintomas de IAM foi fator determinante para a procura de atendimento especializado em tempo hábil(13). Neste estudo, a demora pelo atendimento esteve relacionada ao não reconhecimento dos sinais e sintomas como cardiológicos.

Considerando o estado civil, este estudo apresentou mais de $60 \%$ dos participantes casados, indo ao encontro de resultados encontrados em estudos similares ${ }^{(9-10)}$. A procedência da região metropolitana, ou seja, próximo à instituição hospitalar, aparece em outros estudos ${ }^{(9,11)}$ podendo estar relacionado com a facilidade de acesso ao serviço de alta complexidade.

A procura pela instituição hospitalar, em casos de intercorrências de saúde, neste estudo mostrou estar relacionada com a dificuldade no acesso às consultas com o clínico geral na UBS e com especialista na média complexidade. Estudo que investigou as barreiras ao acesso à APS, como ausência de médico nas UBS e insuficiente oferta de consultas e exames especializados, mostrou que estas tendem a se acentuar nos serviços de maior tecnologia, adiando o atendimento ao usuário e agravando seu quadro de doença ${ }^{(5)}$. Percebe-se que a APS apresenta dificuldade em desempenhar seu papel de ordenadora da atenção à saúde, de continuidade do cuidado com resolutividade, sendo necessário consolidar formas de diálogo entre os profissionais dos diferentes serviços no SUS ${ }^{(14)}$.

O enfoque dos sistemas de saúde na agudização das doenças e não na cronicidade das mesmas também pode ser apontado como motivo para a busca ao serviço de alta complexidade ${ }^{(15)}$. Normalmente o usuário costuma procurar as emergências médicas quando considera que o problema de saúde é grave ou quando avalia que a APS não irá resolver seu problema de saúde ${ }^{(16)}$.

No Brasil, estudos realizados com pacientes submetidos a CRM mostram que mais de 50\% dos participantes apresentaram patologias pré-existentes, sendo as de maior distribuição a HAS e o DM ${ }^{(12,17-}$ ${ }^{18)}$. Estudos similares mostraram que mais da metade dos participantes com insuficiência coronariana 
submetidos a CRM apresentam fatores de risco para DCV, sendo a pressão arterial elevada a mais prevalente, seguido da dislipidemia, DM, tabagismo ${ }^{(9,19-20)}$ e história familiar para $\mathrm{DCV}^{(17,20)}$.

Assim, o acompanhamento de saúde é importante para a prevenção e controle dos fatores de riscos das DCV e comorbidades associadas, por meio da implementação de ações interventivas integradas e intersetoriais de promoção da saúde e prevenção da DCV, de forma individual e coletiva, para auxiliar as pessoas a modificarem os comportamentos de risco, favorecendo a adoção de hábitos de vida saudáveis ${ }^{(21)}$. A atuação dos profissionais de saúde pode contribuir, ou não, na adesão de práticas que atuem no controle de fatores de risco pelos pacientes, como a qualidade nutricional, o sedentarismo, o estresse, a obesidade, com controle qualificado da condição clínica da doença ${ }^{(22)}$.

Apesar do aumento no acesso aos serviços de saúde, dados sobre a assistência farmacêutica mostram que em apenas $45 \%$ dos atendimentos com prescrição, feitos pelo SUS, os pacientes receberam $100 \%$ dos medicamentos prescritos ${ }^{(23)}$. A disponibilidade média de medicamentos na APS no Brasil é de $58,5 \%$, sendo considerada baixa ${ }^{(24)}$, evidenciada por estudo que mostrou que mais de $50 \%$ da população retira medicamento nas farmácias do serviço público de saúde ${ }^{(25)}$. A falta de medicamentos na APS é a principal causa de insatisfação do usuário e fator que interfere no acesso ao serviço de saúde ${ }^{(26)}$. A baixa adesão ao tratamento medicamentoso para DCV está diretamente relacionada às altas taxas de readmissão hospitalar e de mortalidade ${ }^{(27)}$.

Estudo apresentou que 18\% dos pacientes no pré-operatório para CRM já haviam realizado CRM prévia e $12 \%$ intervenção percutânea prévia ${ }^{(9)}$. A adesão a grupos de reabilitação cardíaca no geral é baixa, tal como mostrou este estudo, evidenciando a necessidade de estímulo e incentivo da participação de pacientes que realizaram CRM, como também de pacientes com fatores de risco para $\mathrm{DAC}^{(28)}$.

Neste sentido, a educação em saúde, a oferta de atividades que promovam saúde ou diminuam riscos de doenças e agravos, com acompanhamento da equipe multiprofissional na APS, são indispensáveis. Os grupos de apoio visam à promoção da saúde com foco na melhoria da qualidade de vida, troca de experiências e construção de vínculos, no entanto, por vezes não são oferecidos por falta de infraestrutura, profissionais e outras demandas, considerando ainda o foco curativista do sistema de saúde ${ }^{(29)}$.

O estudo apresentou como limitação o fato de ter sido desenvolvido em uma realidade específica de um estado do sul do Brasil, assim, os achados não podem ser generalizados, carecendo de estudos que investiguem o perfil e acesso da população submetida a CRM em outros locais.

\section{CONCLUSÃO}

O perfil epidemiológico foi constituído por homens, casados, aposentados, procedentes da região metropolitana do Estado, média de idade de 61,3 anos e seis anos de estudo. A hipertensão arterial sistêmica foi a comorbidade com maior expressão seguida da diabetes mellitus, e os principais fatores de risco foram consequentemente a pressão arterial aumentada e a glicemia elevada.

Referente ao acesso aos serviços no SUS para promoção da saúde, prevenção, tratamento e reabilitação da DCV, prevaleceu como porta de entrada no SUS o serviço de emergência da instituição hospitalar diante do quadro agudo da DAC, a dificuldade de acesso às consultas com clínico geral na APS e especialista na média complexidade. Embora a maioria dos participantes revele utilizar a APS para consultas médicas e exames diagnósticos, a falta de medicamentos e grupos terapêuticos no pós-operatório aparece como fatores que dificultam o acesso e acompanhamento de saúde dos participantes neste cenário.

Tais resultados fornecem subsídios para a prática profissional do enfermeiro e demais profissionais de saúde, em especial no âmbito da APS, no qual se observa a necessidade de ações estratégicas que facilitem o acesso, em tempo hábil, aos serviços para acompanhamento de saúde, considerando o perfil epidemiológico desta população e a necessidade de identificação precoce dos fatores de risco e diagnóstico de DAC, assim como o acesso a práticas de reabilitação cardiovascular após a CRM, as quais necessitam ser exploradas neste cenário. 


\section{APOIO FINANCEIRO}

Este estudo recebeu suporte financeiro da Fundação de Amparo a Pesquisa e Inovação do Estado de Santa Catarina (FAPESC) por meio do processo n. 1459/2012.

\section{REFERÊNCIAS}

1. Stipp MAC. A Gerência do cuidado na enfermagem cardiovascular. Esc. Anna Nery. [Internet] 2012;16(1) [acesso em 10 nov 2014]. Disponível: http://dx.doi.org/10.1590/S1414-81452012000100001.

2. da Costa CFS, Vaghetti HH, Santos SSC, Francioni FF, Kerber NPC. A complexidade da rede de atenção à saúde. Cienc Cuid Saude. [Internet] 2015;14(4) [acesso em 15 nov 2016]. Disponível: http://dx.doi.org/10.4025/ cienccuidsaude.v14i4.27791.

3. Barbiani R, Junges JR, Nora CRD, Asquidamini F. A produção científica sobre acesso no âmbito do Sistema Único de Saúde do Brasil: avanços, limites e desafios. Saude soc. [Internet] 2014;23(3) [acesso em 15 nov 2015]. Disponível: http://dx.doi.org/10.1590/S0104-12902014000300010.

4. Balasubramanian H, BiehI S, Dai L, Muriel A. Dynamic allocation of same-day requests in multiphysician primary care practices in the presence of prescheduled appointments. Health Care Manag Sci. [Internet] 2014;17(1) [acesso em 15 nov 2015]. Disponível: http://dx.doi.org/10.1007/s10729-013-9242-2.

5. Sousa FOS, de Medeiros KR, Gurgel Júnior GD, de Albuquerque PC. Do normativo à realidade do Sistema Único de Saúde: revelando barreiras de acesso na rede de cuidados assistenciais. Ciênc. saúde coletiva. [Internet] 2014;19(4) [acesso em 15 nov 2015]. Disponível: http://dx.doi.org/10.1590/1413-81232014194.01702013.

6. Grden CRB, Weise T, Reche PM, Borges PKO, Cabral LPA. Características sociodemográficas e de acesso de longevos aos serviços de saúde. Cienc. Cuid. Saude. [Internet] 2015;14(4) [acesso em 15 nov 2016]. Disponível: http://dx.doi.org/10.4025/cienccuidsaude.v14i4.24985.

7. Stein-Backes D, Stein-Backes M, Lorenzini-Erdmann A, Büscher A, Salazar-Maya MA. Significado da prática social do enfermeiro com e a partir do Sistema Único de Saúde brasileiro. Aquichán. [Internet] 2014;14(4) [acesso em 15 nov 2016]. Disponível: http://dx.doi.org/10.5294/aqui.2014.14.4.10.

8. Minayo MCS. O desafio do conhecimento: pesquisa qualitativa em saúde. 13a ed. São Paulo: Hucitec; 2013.

9. de Oliveira RM, de Sena ZGF, Frota LMCP, de Oliveira JBB. Quality of life of women submitted to myocardial revascularization surgery in a public hospital. Rev. bras. promoç. Saúde. [Internet] 2010;23(3) [acesso em 15 nov 2016]. Disponível: http://dx.doi.org/10.5020/18061230.2010.p237.

10. Ponte KMA, da Silva LF, Borges MCLA, Aragão AEA, Arruda LP, de Galiza FT. Caracterização de pessoas em cirurgias cardíacas: estudo descritivo. Rev. pesqui. cuid. fundam. [Internet] 2013;5(2) [acesso em 15 nov 2016]. Disponível: http://dx.doi.org/10.9789/2175-5361.2013v5n2p3677.

11. Baggio MA, Parizoto GM, Callegaro GD, Koerich C, Erdmann AL. Incidência e caraterísticas sociodemográficas de pacientes internados com coronariopatia. Rev. Enf. Ref. [Internet] 2011;serllI(5) [acesso em 15 nov 2016]. Disponível: http://dx.doi.org/10.12707/RIII1037.

12. Assis CC, Lopes JL, Nogueira-Martins LA, de Barros ALBL. Acolhimento e sintomas de ansiedade em pacientes no pré-operatório de cirurgia cardíaca. Rev. bras. enferm. [Internet] 2014;67(3) [acesso em 15 nov 2016]. Disponível: http://dx.doi.org/ 10.5935/0034-7167.20140053.

13. Bastos AS, Beccaria LM, Contrin LM, Cesarino CB. Tempo de chegada do paciente com infarto agudo do miocárdio em unidade de emergência. Rev Bras Cir Cardiovasc. [Internet] 2012;27(3) [acesso em 15 nov 2016]. Disponível: http://dx.doi.org/10.5935/1678-9741.20120070.

14. de Albuquerque MSV, Lyra TM, Farias SF, Mendes MFM, Martelli PJL. Acessibilidade aos serviços de saúde: uma análise a partir da Atenção Básica em Pernambuco. Saúde debate. [Internet] 2014;38(n.esp) [acesso em 15 nov 2016]. Disponível: http://dx.doi.org/10.5935/0103-1104.2014S014. 
15. Mendes EV. As redes de atenção à saúde. Ciênc. saúde coletiva. [Internet] 2010;15(5) [acesso em 15 nov 2016$].$ Disponível: http://dx.doi.org/10.1590/S1413-81232010000500005.

16. Pires MRGM, Göttems LBD, Cupertino TV, Leite LS, do Vale LR, de Castro MA, et al. A Utilização dos serviços de atenção básica e de urgência no SUS de Belo Horizonte: problema de saúde, procedimentos e escolha dos serviços. Saúde Soc. [Internet] 2013;22(1) [acesso em 15 nov 2016]. Disponível: http://dx.doi.org/10.1590/S010412902013000100019.

17. de Sousa AG, Fichino MZS, da Silva GS, Bastos FCC, Piotto RF. Epidemiology of coronary artery bypass grafting at the Hospital Beneficência Portuguesa, São Paulo. Rev Bras Cir Cardiovasc. [Internet] 2015;30(1) [acesso em 15 nov 2016]. Disponível: http://dx.doi.org/10.5935/1678-9741.20140062.

18. Xavier TT, Torres GV, dos Reis LA, da Silva RAR, Costa IKF, Mendes FRP. Avaliação de saúde e da dor no pósoperatório de idosos submetidos à cirurgia cardíaca. Texto Contexto Enferm. [Internet] 2011;20(n.esp) [acesso em 15 nov 2016]. Disponível: http://dx.doi.org/10.1590/S0104-07072011000500029.

19. Gimenes C, Barrile SR, Martinelli B, Ronchi CF, Arca EA, Gimenes R, et al. Association of pre and intraoperative variables with postoperative complications in coronary artery bypass graft surgery. Rev Bras Cir Cardiovasc. [Internet] 2013;28(4) [acesso em 15 nov 2016]. Disponível: http://dx.doi.org/10.5935/1678-9741.20130084.

20. Tonial R, Moreira DM. Perfil clínico-epidemiológico dos pacientes submetidos à cirurgia de revascularização do miocárdio no instituto de cardiologia de Santa Catarina, São José - SC. Arquivos Catarinenses de Medicina. [Internet] 2011;40(4) [acesso em 15 nov 2016]. Disponível: http://www.acm.org.br/revista/pdf/artigos/894.pdf.

21. Magalhães FJ, Mendonça LBA, Rebouças CBA, Lima FET, Custódio IL, de Oliveira SC. Fatores de risco para doenças cardiovasculares em profissionais de enfermagem: estratégias de promoção da saúde. Rev. bras. enferm. [Internet] 2014;67(3) [acesso em 15 nov 2016]. Disponível: http://dx.doi.org/10.5935/0034-7167.20140052.

22. Costa YF, de Araújo OC, de Almeida LBM, Viegas SMF. Educational role of nurses in joining the treatment of Systemic Arterial Hypertension: integrative literature review. O Mundo da Saúde. [Internet] 2014;38(4) [acesso em 15 nov 2016]. Disponível: http://dx.doi.org/10.15343/0104-7809.20143804473481.

23. Viacava F. Dez anos de informação sobre acesso e uso de serviços de saúde. Cad. Saúde Pública. [Internet] 2010;26(12) [acesso em 15 nov 2016]. Disponível: http://dx.doi.org/10.1590/S0102-311X2010001200001.

24. Mendes LV, Campos MR, Chaves GC, da Silva RM, Freitas PS, Costa KS, et al. Disponibilidade de medicamentos nas unidades básicas de saúde e fatores relacionados: uma abordagem transversal. Saúde debate. [Internet] 2014;38(n.esp) [acesso em 15 nov 2016]. Disponível: http://dx.doi.org/10.5935/0103-1104.2014S009.

25. Bezerra TA, de Brito MAA, Costa KNFM. Caracterização do uso de medicamentos entre idosos atendidos em uma unidade básica de saúde da família. Cogitare Enferm. [Internet] 2016;21(1) [acesso em 15 nov 2016]. Disponível: http://dx.doi.org/10.5380/ce.v21i1.43011.

26. Mendes ACG, Miranda GMD, Figueiredo KEG, Duarte PO, Furtado BMASM. Acessibilidade aos serviços básicos de saúde: um caminho ainda a percorrer. Ciênc. saúde coletiva. [Internet] 2012;17(11) [acesso em 15 nov 2016]. Disponível: http://dx.doi.org/10.1590/S1413-81232012001100007.

27. Chowdhury R, Khan H, Heydon E, Shroufi A, Fahimi S, Moore C, et al. Adherence to cardiovascular therapy: a meta-analysis of prevalence and clinical consequences. Eur Heart J. [Internet] 2013;34(38) [acesso em 15 nov 2016]. Disponível: http://doi.org/10.1093/eurheartj/eht295.

28. Aikawa P, Cintra ARS, de Oliveira Júnior AS, da Silva CTM, Pierucci JD, Afonso MS et al. Reabilitação cardíaca em pacientes submetidos a cirurgia de revascularização do miocárdio. Rev Bras Med Esporte. [Internet] 2014;20(1) [acesso em 15 nov 2016]. Disponível: http://dx.doi.org/10.1590/S1517-86922014000100011.

29. Maceno PR, Heidemann ITSB. Desvelando as ações dos enfermeiros nos grupos da atenção primária à saúde. Texto Contexto Enferm. [Internet] 2016;25(4) [acesso em 02 fev 2017]. Disponível: http://dx.doi.org/10.1590/010407072016002140015. 